\title{
DpenPhos/Rh(I)催化的 $\beta$-脱氢氨基酸酯的不对称氢化反应
}

\author{
刘㰍 王正 丁奎岭* \\ (中国科学院上海有机化学研究所 金属有机化学国家重点实验室 上海 200032)
}

\begin{abstract}
摘要 本文研究了一类结构可调手性单齿亚磷酰胺配体 DpenPhos 在 $\mathrm{Rh}(\mathrm{I})$ 催化的 $E$ 和 $Z$ 型 $\beta$-脱氢氨基酸酯的不对称催 化氢化反应中的应用. 经过系统的反应条件和配体结构优化, 发现 $\mathrm{N}$ 原子上含有 $\mathrm{H}$ 的亚磷酰胺配体与 $\mathrm{Rh}(\mathrm{I})$ 形成的催化 剂通常比 $\mathrm{N}$ 原子上不含 $\mathrm{H}$ 的配体表现出更高的反应活性. 在 $E$ 型 $\beta$-脱氢氨基酸酯的不对称氢化反应中, 催化剂 $(R, R)-$ $3 \mathrm{k} / \mathrm{Rh}$ (I)表现突出，可以实现底物的常压催化氢化，取得了 $92 \% \sim 96 \%$ 的对映选择性，催化剂用量可降低至 $0.2 \mathrm{~mol} \%$; 对于 $Z$ 型 $\beta$-脱氢氨基酸酯的不对称氢化反应, 则 $(R, R)-3 \mathrm{l} / \mathrm{Rh}(\mathrm{I})$ 为最优催化剂, 可以获得 $92 \% \sim 98 \% e e$ 值的氢化产物, 特 别是对于 $\beta$-芳基取代衍生物的氢化反应，相应氢化产物的 $e e$ 值可以达到 96\% 98\%. 该类催化剂为天然或非天然光学 活性 $\beta$ 氨基酸的合成提供了一个简便、高效的方法.

关键词 不对称催化; 氢化; 单磷配体; 亚磷酰胺; 铑; $\beta$-脱氢氨基酸甲酯
\end{abstract}

\section{DpenPhos/Rh(I) Catalyzed Asymmetric Hydrogenation of Dehydro- $\beta$-Amino Acid Esters}

\author{
Liu, Yan Wang, Zheng Ding, Kuiling* \\ (State Key Laboratory of Organometallic Chemistry, Shanghai Institute of Organic Chemistry, Chinese Academy of Sciences, \\ Shanghai 200032)
}

\begin{abstract}
Enantiomerically pure $\beta$-amino acids and their derivatives are very important chiral building blocks for the synthesis of $\beta$-peptides, $\beta$-lactams, and many important biologically active compounds. Among various methods developed for their synthesis, catalytic asymmetric hydrogenation of the corresponding dehydro- $\beta$-amino acid esters represents one of the most efficient and environmentally benign approaches, in which the Rh(I) catalysts containing chiral phosphorous ligands play a central role. Although a variety of Rh(I) complexes of bidentate or monodentate ligands have been discovered for asymmetric hydrogenation of dehydro- $\beta$-amino acid esters, some challenging issues still remain in terms of efficiency, enantioselectivity, and substrate scope. In the present work, a variety of $\mathrm{Rh}(\mathrm{I})$ complexes of modular monodentate phosphoramidite ligands, DpenPhos, have been systematically investigated for the asymmetric hydrogenation of $(E)$ - or $(Z)$ - $\beta$-substituted dehydro- $\beta$-amino acid esters. It was found that the presence of a $\mathrm{N}-\mathrm{H}$ moiety in the phosphoramidite ligand is critically important for achieving high activity of the catalysis. Both $(Z)$ - and $(E)$-geometrical isomers of the $\beta$-acetamido acrylic acid esters can be hydrogenated in the presence of DpenPhos/Rh(I) catalysts to form the same enantiomers, albeit slightly different conditions were required to attain optimal enantioselectivities. $(R, R)-3 \mathbf{k} / \mathrm{Rh}(\mathrm{I})(1 \mathrm{~mol} \%)$ was disclosed to be optimal for the hydrogenation of $(E)$ - $\beta$-alkyl substituted dehydro- $\beta$-amino acid esters in dichloromethane under a relatively low pressure of hydrogen $(0.5 \mathrm{MPa})$, affording the corresponding $\beta$-amino acid esters with $92 \%-96 \%$ ee. The reaction still proceeded smoothly without significant sacrifice of activity or enantioselectivity either at a catalyst loading of $0.2 \mathrm{~mol} \%$ or $0.1 \mathrm{MPa}$ pressure of $\mathrm{H}_{2}$. For the hydrogenation of more challenging $(Z)-\beta$-alkyl or aryl substituted dehydro- $\beta$-amino acid esters, $(R, R)-3 \mathrm{l} / \mathrm{Rh}(\mathrm{I})$ in combination with the dichloromethane/isopropanol $(V / V=2 / 1)$ mixed solvent system turned out to be the best in terms of enantioselectivity and catalytic activity, giving a variety of $\beta$-alkyl or aryl substituted $\beta$-amino acid esters in $>99 \%$ yields with excellent optical purities $(92 \%-98 \% e e)$.
\end{abstract}

Keywords asymmetric catalysis; hydrogenation; monodentate ligand; phosphoramidite; rhodium; dehydro- $\beta$-amino acid ester

\section{1 引言}

在不对称催化氢化研究领域, 尽管第一个均相催化 的例子是采用手性单膦配体与 $\mathrm{Rh}(\mathrm{I})$ 形成的催化剂, 但
在公元 2000 年以前, 应用手性双磷配体几乎主导了不 对称催化氢化研究领域 ${ }^{[1]}$. 进入 21 世纪, 在 Pringle, Reetz 和 Feringa 等的开创性工作中 ${ }^{[2]}$, 发现在铑催化的 不对称氢化反应中，手性单齿亚磷酸酯或亚磷酰胺类配

\footnotetext{
*E-mail: kding@mail.sioc.ac.cn

Received March 23, 2012; published May 3, 2012.

Supporting information for this article is available free of charge via the Internet at http://sioc-journal.cn. (No. 2010CB833300).

项目受国家自然科学基金(Nos. 21172237, 21121062, 21032007)和“973 计划” (No. 2010CB833300)资助.
}

Project supported by the National Natural Science Foundation of China (Nos. 21172237, 21121062, 21032007) and Major Basic Research Program of China 
体表现出可以与双磷配体相媲美甚至更好的对映选择 性控制，从而在概念上突破了只有手性双齿磷配体才能 获得高对映选择性的传统观念, 进而引发了手性单磷配 体在不对称催化反应特别是在不对称催化氢化反应中 应用的复兴 ${ }^{[3]}$. 一些代表性的手性单膦配体 1 3 (Mo$\operatorname{noPhos}^{[2 \mathrm{~b}]}, \mathrm{SiPhos}^{[4]}$ 和 DpenPhos ${ }^{[5]}$ )如 Figure 1 所示, 这些 配体的特点是合成简单, 原料价廉易得, 与金属形成的 催化剂在反应中有足够的稳定性和高的反应活性与对 映选择性.
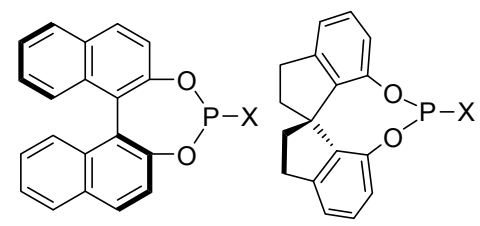

2a $X=O R$

1a $X=O R$

1b $X=$ alkyl or aryl

2b $X=$ alkyl or aryl

1c $X=\mathrm{NR}_{2}$, MonoPhos $2 \mathrm{c} X=\mathrm{NR}_{2}$, SIPHOS

图 1 一些代表性的手性单磷配体

Figure 1 Some representative monodentate phosphorus ligands

最近我们课题组报道了一类基于 1,2-二苯基乙二胺 骨架的手性单磷配体 DpenPhos ${ }^{[5]}$ (3), 其在 Rh(I)催化的 $\alpha$-脱氢氨基酸酯衍生物、芳基烯胺衍生物、 $\alpha$-乙酰氧基 丙烯酸酯衍生物、芳基取代衣康酸酯、 $\alpha$-或 $\beta$-酰氨基不 饱和膦酸酯以及 $\alpha$-或 $\beta$-酰氧基不饱和膦酸酯等衍生物 的不对称催化氢化反应中(Figure 2$)^{[5 \mathrm{a} \sim 5 \mathrm{~d}]}$, 表现出了优 秀的对映选择性, 同时发现, DpenPhos 配体骨架上取代 基 R 对反应的选择性具有很好的调节作用, 而亚磷酰胺 $\mathrm{N}$ 原子上的取代基 $\mathrm{R}^{\prime}$ 或 $\mathrm{R}^{\prime \prime}$ 对反应的活性有重要影响, 特别是当 $R^{\prime}$ 或 $R^{\prime \prime}$ 为 $H$ 时, 催化反应活性明显提高, 使得 一些对于使用双膦或者其它单磷配体难以实现的底物 的氢化反应得以高效进行, 通过对催化剂核磁共振波谱

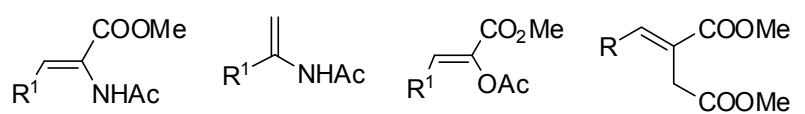

$96 \% \sim>99 \%$ ee $96 \% \sim>99 \%$ ee $96 \% \sim>99 \%$ ee $96 \% \sim>99 \%$ ee
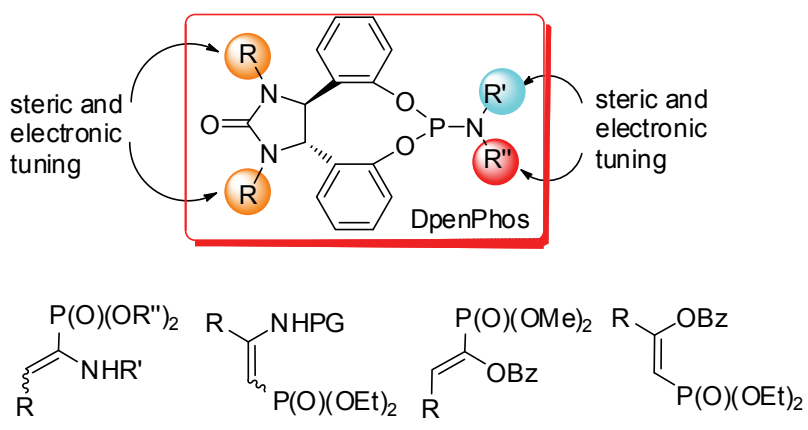

$86 \% \sim>99 \%$ ee $81 \% \sim>99 \%$ ee $90 \% \sim>99 \%$ ee $93 \% \sim 96 \%$ ee

图 2 DpenPhos/Rh(I)催化的一些烯烃衍生物的氢化反应.

Figure 2 DpenPhos/Rh(I) catalyzed asymmetric hydrogenation of some olefinic substrates.
和单晶衍射研究表明，这可能与催化剂中两个单磷配体 之间存在某种形式氢键作用有关 ${ }^{[5 \mathrm{~b} \sim 5 \mathrm{~d}]}$. 在上述工作基 础上，本文继续探索此类配体在铑催化的 $\beta$-脱氢氨基酸 酯衍生物催化氢化中的应用，结果发现在优化的反应条 件下, 对于 $(E)$ 或 $(Z)-\beta$-脱氢氨基酸甲酯衍生物的不对称 催化氢化，可以获得 $92 \% \sim 98 \%$ 的对映选择性，催化剂 用量最低可以降至 $0.2 \mathrm{~mol} \%$.

\section{2 结果与讨论}

光学纯 $\beta$-氨基酸及其衍生物是很多天然产物和药物 的重要结构单元 ${ }^{[6]}$, 因此关于 $\beta$-氨基酸及其衍生物不对 称合成方法的研究受到广泛关注，在各种合成方法中 ${ }^{[7]}$, 通过 $\beta$-脱氢氨基酸及其衍生物不对称催化氢化是最为 直接、高效和清洁的方法 ${ }^{[7 \sim 10]}$. 由于其前手性化合物 $\beta$ 取代- $\beta$-酰氨基丙烯酸酯结构的特性, 分为 $Z$-异构体和 $E$-异构体， $Z$-异构体上的酰氨基质子与酯基上的羰基氧 可以形成稳定的分子内氢键, 导致在一些催化体系中, 难以取得高的对映选择性和反应活性, 因此在 $\beta$-取代$\beta$-酰氨基丙烯酸酯衍生物的不对称催化氢化中, 仍然存 在一些挑战 ${ }^{[7 \sim 10]}$. 本文将针对上述底物的不对称催化氢 化反应，探索我们发展的 DpenPhos 配体在铑催化的 $\beta$ 脱氢氨基酸酯衍生物催化氢化中的应用.

\subsection{DpenPhos 配体(3a $\sim 30)$ 的合成}

配体 DpenPhos $(\mathbf{3 a} \sim \mathbf{3 o}$, Figure 3) 合成的关键是制备 光学醇 1,2-二(2-甲氧基苯基)乙二胺关键中间体，参考 我们前期的研究工作 ${ }^{[5 \sim 5 c]}$, 从邻甲氧基苯甲醛出发, 经 过四步反应，可以 $41 \%$ 的总收率制得外消旋的 1,2-二(2甲氧基苯基)乙二胺; 运用价廉易得的酒石酸为拆分试 剂, 以 $39 \%$ 和 $45 \%$ 的收率分别得到光学纯 $(S, S)$ 和 $(R, R)-$ 1,2-二(2-甲氧基苯基)乙二胺，再经过成咪唑啉酮环、咪 唑啉酮修饰和脱甲基保护基三步反应，以中等到良好的 收率得到光学纯的二酚化合物，二酚化合物经过一步或 两步转化, 即可以中等到良好的收率获得光学纯配体 3a $\sim 30$.

\subsection{DpenPhos $(\mathbf{3 a} \sim 30)$ 在 $\mathrm{Rh}(\mathrm{I})$ 催化的 $(E)-\beta$-脱氢氨 基酸甲酯衍生物不对称氢化中的应用}

由于 DpenPhos 配体在 $\mathrm{Rh}(\mathrm{I})$ 催化 $\alpha$-脱氢氨基酸酯 衍生物和芳基烯胺衍生物氢化反应中的突出表现 ${ }^{[5 a]}$, 我 们首先尝试这类配体在 $\beta$-脱氢氨基酸甲酯氢化反应中 应用的可能性. 一般认为 $(E)-\beta$-脱氢氨基酸甲酯是在 $\beta$ 脱氢氨基酸甲酯催化不对称氢化中较易进行的一类底 物, 因此首先以 $(E)-\beta$-脱氢氨基酸甲酯衍生物为底物进 行不对称催化氢化的可行性研究.

如表 1 所示，以 $(E)-3$-乙酰胺基-2-丁烯酸甲酯 $(E)-\mathbf{4 a}$ 为模型底物, $\mathrm{Rh}(\mathrm{cod})_{2} \mathrm{BF}_{4}$ 作为催化剂前体, 二氯甲烷为 溶剂, $2 \mathrm{MPa}$ 氢气压力下, $1 \mathrm{~mol} \%$ 催化剂用量下对配体 $(R, R)-\mathbf{3 a} \sim \mathbf{3 h}$ 进行氢化的初步试探工作. 从表中数据可 
<smiles>[R]NP([R])Oc1ccccc1Oc1c([Y])n([R])c(=O)n1[Y]</smiles>

$(R, R)-3 \mathrm{a}: \mathrm{R}^{1}=\mathrm{H}, \mathrm{R}^{2}=\mathrm{CH}_{3}$ $(R, R)-3 \mathbf{b}: \mathrm{R}^{1}=\mathrm{CH}_{3}, \mathrm{R}^{2}=\mathrm{CH}_{3}$ $(R, R)-3 \mathrm{c}: \mathrm{R}^{1}=\mathrm{CH}_{3} \mathrm{CH}_{2}, \mathrm{R}^{2}=\mathrm{CH}_{3}$ $(R, R)-3 \mathrm{~d}: \mathrm{R}^{1}=\mathrm{CH}_{3} \mathrm{CH}_{2}, \mathrm{R}^{2}=\mathrm{CH}_{3} \mathrm{CH}_{2}$ $(R, R)-3 \mathrm{e}: \mathrm{R}^{1}=\mathrm{C}_{6} \mathrm{H}_{5} \mathrm{CH}_{2}, \mathrm{R}^{2}=\mathrm{CH}_{3}$ $(R, R)-3 f: \mathrm{R}^{1}=\mathrm{C}_{6} \mathrm{H}_{5} \mathrm{CH}_{2}, \mathrm{R}^{2}=\mathrm{CH}_{3} \mathrm{CH}_{2}$ $(R, R)-3 \mathrm{~g}: \mathrm{R}^{1}=3,5-\left(\mathrm{CH}_{3}\right)_{2} \mathrm{C}_{6} \mathrm{H}_{3} \mathrm{CH}_{2}, \mathrm{R}^{2}=\mathrm{CH}_{3}$ $(R, R)-3 h: \mathrm{R}^{1}=3,5-\left(t-\mathrm{C}_{4} \mathrm{H}_{9}\right)_{2} \mathrm{C}_{6} \mathrm{H}_{3} \mathrm{CH}_{2}, \mathrm{R}^{2}=\mathrm{CH}_{3}$<smiles>[R]NPOc1ccccc1OP(N[R])n1c(=O)n([R])c(=O)n1[R]</smiles>

(S,S)-3i: $\mathrm{R}^{1}=\mathrm{CH}_{3} \mathrm{CH}_{2}, \mathrm{R}^{2}=\mathrm{CH}_{3} \mathrm{CH}_{2} \mathrm{CH}_{2}$ $\left(\right.$ S,S)-3: $\mathrm{R}^{1}=\mathrm{CH}_{3} \mathrm{CH}_{2}, \mathrm{R}^{2}=\mathrm{C}_{6} \mathrm{H}_{5} \mathrm{CH}_{2}$ $(R, R)-3 \mathbf{k}: \mathrm{R}^{1}=\mathrm{C}_{6} \mathrm{H}_{5} \mathrm{CH}_{2}, \mathrm{R}^{2}=\mathrm{CH}_{3} \mathrm{CH}_{2} \mathrm{CH}_{2}$ $(R, R)^{-31:} \mathrm{R}^{1}=\mathrm{C}_{6} \mathrm{H}_{5} \mathrm{CH}_{2}, \mathrm{R}^{2}=\mathrm{C}_{6} \mathrm{H}_{5} \mathrm{CH}_{2}$ $(\mathrm{S}, \mathrm{S}, \mathrm{R})-3 \mathrm{~m}: \mathrm{R}^{1}=\mathrm{C}_{6} \mathrm{H}_{5} \mathrm{CH}_{2}, \mathrm{R}^{2}=(\mathrm{R})-\mathrm{C}_{6} \mathrm{H}_{5}\left(\mathrm{CH}_{3}\right) \mathrm{CH}$ $(S, S, S)-3 n: \mathrm{R}^{1}=\mathrm{C}_{6} \mathrm{H}_{5} \mathrm{CH}_{2}, \mathrm{R}^{2}=\left(\mathrm{S}^{2}-\mathrm{C}_{6} \mathrm{H}_{5}\left(\mathrm{CH}_{3}\right) \mathrm{CH}\right.$ $(R, R)-3 \mathrm{o}: \mathrm{R}^{1}=3,5-\left(\mathrm{CH}_{3}\right)_{2} \mathrm{C}_{6} \mathrm{H}_{3} \mathrm{CH}_{2}, \mathrm{R}^{2}=\mathrm{CH}_{3} \mathrm{CH}_{2} \mathrm{CH}_{2}$

图 3 本研究中所用的 DpenPhos 配体

Figure 3 DpenPhos ligands employed in the present study

以看出, 初步实验结果并不理想, 在所尝试的几种配体 中(Entries 1 8), 除了 $(R, R)-\mathbf{3 f}$ 之外, 其余的配体和铑形 成的催化剂活性都很低, 而使用配体 $(R, R)-3 \mathrm{f}$ 也只取得 了中等的 $e e$ 值(Entry 6), 但通过对 $(R, R)$-3f 的结构进行 分析, 发现与 $\mathrm{P}$ 相连 $\mathrm{N}$ 上的取代基对反应的活性及对映 选择性有很大的影响, 可能因为与 $\mathrm{P}$ 相连 $\mathrm{N}$ 上的取代基 距离反应活性中心较近, 如果我们在此位置上对配体进 行改造, 或许会取得满意的结果.

我们前期的研究结果表明 ${ }^{[5 b}$-5d], 当 DpenPhos 配体 中 $\mathrm{R}^{\prime}$ 或 $\mathrm{R}^{\prime \prime}$ (图 2)为 $\mathrm{H}$ 时, 相应的 $\mathrm{Rh}(\mathrm{I})$ 络合物的催化活性 明显提高. 因此进一步尝试与 $\mathrm{P}$ 相连 $\mathrm{N}$ 上含有 $\mathrm{H}$ 的配体 在此反应体系中的应用, 首先用 $(S, S, R)-\mathbf{3} \mathrm{m}$ 为配体, 在 上述相同反应条件下对 $(E)$-3-乙酰氨基-2-丁烯酸甲酯 (E)-4a 进行氢化反应尝试, 发现氢化反应可以顺利进行, 以定量的原料转化和 $89 \%$ 的 ee 值获得氢化反应产物 (Entry 9). 氢气压力的变化对于 $(E)-\mathbf{4 a}$ 的不对称催化氢 化中的对映选择性有一定影响(Entries 9 11), 将氢气 压力降为 $0.5 \mathrm{MPa}$, 反应的对映选择性不受影响(Entry 10 ); 继续降低压力至 $0.1 \mathrm{MPa}$, 虽然产物的 $e e$ 值有所下 降, 但原料转化仍可达到 100\% (Entry 11), 显示了很高 的催化活性.

接下来进一步考察了底物浓度的变化对反应的影 响(Entries 10,12 14), 发现在 $0.5 \mathrm{MPa}$ 氢气压力下, 随
表 1 DpenPhos $(\mathbf{3 a} \sim 3 \mathbf{3 o})$ 在 $\mathrm{Rh}(\mathrm{I})$ 催化的 $(E)-\mathbf{4 a}$ 的不对称氢化中的应 用: 配体与反应条件优化.

Table 1 Application of DpenPhos (3a $\sim 30)$ in Rh(I)-catalyzed asymmetric hydrogenation of $(E)-\beta$-dehydroamino acid ester $(E)-\mathbf{4 a}$ : chiral ligand and reaction condition optimization.

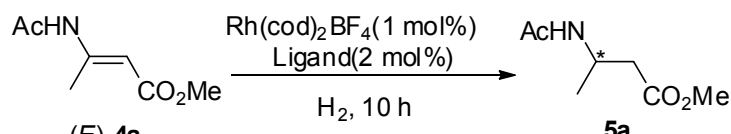

\begin{tabular}{|c|c|c|c|c|c|c|}
\hline Entry & Ligand $I$ & $P\left(\mathrm{H}_{2}\right) / \mathrm{MPa}$ & Solvent & $\begin{array}{c}(E)-\mathbf{4 a}] / \\
(\mathrm{mol} / \mathrm{L})\end{array}$ & $\begin{array}{c}\text { Conv }^{a} \\
1 \% \\
\end{array}$ & $\begin{array}{c}e e^{b} / \% \\
\text { (config. }{ }^{\text {) }}\end{array}$ \\
\hline 1 & $(R, R)-\mathbf{3 a}$ & 2 & $\mathrm{CH}_{2} \mathrm{Cl}_{2}$ & 0.16 & Trace & n.d. \\
\hline 2 & $(R, R)-\mathbf{3 b}$ & 2 & $\mathrm{CH}_{2} \mathrm{Cl}_{2}$ & 0.16 & Trace & n.d. \\
\hline 3 & $(R, R)-\mathbf{3 c}$ & 2 & $\mathrm{CH}_{2} \mathrm{Cl}_{2}$ & 0.16 & Trace & n.d. \\
\hline 4 & $(R, R)-\mathbf{3 d}$ & 2 & $\mathrm{CH}_{2} \mathrm{Cl}_{2}$ & 0.16 & Trace & n.d. \\
\hline 5 & $(R, R)-\mathbf{3 e}$ & 2 & $\mathrm{CH}_{2} \mathrm{Cl}_{2}$ & 0.16 & Trace & n.d. \\
\hline 6 & $(R, R)-\mathbf{3 f}$ & 2 & $\mathrm{CH}_{2} \mathrm{Cl}_{2}$ & 0.16 & 100 & $51(S)$ \\
\hline 7 & $(R, R)-\mathbf{3 g}$ & 2 & $\mathrm{CH}_{2} \mathrm{Cl}_{2}$ & 0.16 & Trace & n.d. \\
\hline 8 & $(R, R)-\mathbf{3 h}$ & 2 & $\mathrm{CH}_{2} \mathrm{Cl}_{2}$ & 0.16 & Trace & n.d. \\
\hline 9 & $(S, S, R)-\mathbf{3} \mathbf{m}$ & 2 & $\mathrm{CH}_{2} \mathrm{Cl}_{2}$ & 0.16 & $>99$ & $89(R)$ \\
\hline 10 & $(S, S, R)-\mathbf{3} \mathbf{m}$ & 0.5 & $\mathrm{CH}_{2} \mathrm{Cl}_{2}$ & 0.16 & $>99$ & $89(R)$ \\
\hline 11 & $(S, S, R)-\mathbf{3} \mathbf{m}$ & 0.1 & $\mathrm{CH}_{2} \mathrm{Cl}_{2}$ & 0.16 & $>99$ & $81(R)$ \\
\hline 12 & $(S, S, R)-\mathbf{3} \mathbf{m}$ & 0.5 & $\mathrm{CH}_{2} \mathrm{Cl}_{2}$ & 0.33 & $>99$ & $86(R)$ \\
\hline 13 & $(S, S, R)-\mathbf{3} \mathbf{m}$ & 0.5 & $\mathrm{CH}_{2} \mathrm{Cl}_{2}$ & 0.08 & $>99$ & $91(R)$ \\
\hline 14 & $(S, S, R)-\mathbf{3} \mathbf{m}$ & 0.5 & $\mathrm{CH}_{2} \mathrm{Cl}_{2}$ & 0.05 & $>99$ & $89(R)$ \\
\hline 15 & $(S, S, R)-\mathbf{3} \mathbf{m}$ & 0.5 & THF & 0.08 & Trace & n.d. \\
\hline 16 & $(S, S, R)-\mathbf{3} \mathbf{m}$ & 0.5 & Toluene & 0.08 & Trace & n.d. \\
\hline 17 & $(S, S, R)-\mathbf{3} \mathbf{m}$ & 0.5 & $\mathrm{ClCH}_{2} \mathrm{CH}_{2} \mathrm{Cl}$ & $\begin{array}{ll}1 & 0.08\end{array}$ & $>99$ & $89(R)$ \\
\hline 18 & $(S, S)-\mathbf{3 i}$ & 0.5 & $\mathrm{CH}_{2} \mathrm{Cl}_{2}$ & 0.08 & $>99$ & $89(R)$ \\
\hline 19 & $(S, S)-\mathbf{3} \mathbf{j}$ & 0.5 & $\mathrm{CH}_{2} \mathrm{Cl}_{2}$ & 0.08 & $>99$ & $89(R)$ \\
\hline 20 & $(R, R)-\mathbf{3 k}$ & 0.5 & $\mathrm{CH}_{2} \mathrm{Cl}_{2}$ & 0.08 & $>99$ & $96(S)$ \\
\hline 21 & $(R, R)-\mathbf{3} \mathbf{I}$ & 0.5 & $\mathrm{CH}_{2} \mathrm{Cl}_{2}$ & 0.08 & $>99$ & $94(S)$ \\
\hline 22 & $(S, S, S)-\mathbf{3 n}$ & 0.5 & $\mathrm{CH}_{2} \mathrm{Cl}_{2}$ & 0.08 & $>99$ & $83(R)$ \\
\hline 23 & $(R, R)-\mathbf{3 o}$ & 0.5 & $\mathrm{CH}_{2} \mathrm{Cl}_{2}$ & 0.08 & $>99$ & $92(S)$ \\
\hline
\end{tabular}

${ }^{a}$ Determined by ${ }^{1} \mathrm{H}$ NMR. ${ }^{b}$ Determined by chiral GC. ${ }^{c}$ Absolute configuration was determined by comparison of $[\alpha]_{\mathrm{D}}^{20}$ with that reported in literature.

着底物浓度的降低, 产物的 $e e$ 值有增高的趋势, 这可能 是因为在浓度较高的条件下, 脱氢氨基酸酯和配体形成 氢键，从而改变了部分催化剂的结构. 当底物的浓度为 $0.08 \mathrm{~mol} / \mathrm{L}$ 时，产物的 $e e$ 值可以达到 $91 \%$ (Entry 13)，底 物的浓度进一步降低至 $0.05 \mathrm{~mol} / \mathrm{L}$ 时, 虽然也得到定量 的转化, 但产物的 $e e$ 值降低为 $89 \%$ (Entry 14). 溶剂效 应研究表明, 催化反应在二氯甲烷和 1,2-二氯乙烷中都 可以取得较好的结果(Entries 13,15 17). 因此优化的 反应条件为二氯甲烷为溶剂, $0.5 \mathrm{MPa}$ 氢气压力, 底物浓 度为 $0.08 \mathrm{~mol} / \mathrm{L}$ (Entry 13 ).

在优化的反应条件下，我们对其它 $\mathrm{N}$ 原子上含有 $\mathrm{H}$ 的配体 3i 3o 进行了篮选(Table 1, Entries 18 23), 实 验结果表明, 这些配体与 $\mathrm{Rh}(\mathrm{I})$ 的络合物都是非常有效 的催化剂, 在 $(E)-\mathbf{4 a}$ 的催化氢化中都可以取得定量的底 
物转化和良好到优秀的对映选择性( $83 \% \sim 96 \% e e)$, 很 显然产物的绝对构型由配体骨架的手性决定, 而配体中 取代基 $\mathrm{R}^{1}$ 和 $\mathrm{R}^{2}$ 对催化反应的活性影响不大，但对反应 的对映选择性有一定调节作用, 特别是配体中与 $\mathrm{N}$ 原子 相连碳原子的手性对反应的对映选择性有明显影响 (Entries 13 vs 22), 其中使用配体 $(R, R)-3 \mathbf{k}$ 取得最好的结 果, 氢化产物 $5 \mathbf{a}$ 对映异构体过量达到 $96 \%$.

接下来进行底物的拓展, 在制备 $\beta$-芳基- $\beta$-酰氨基 丙烯酸甲酯时, 由于 $E$ 型异构体可转化为更加稳定的 $Z$ 型异构体(由于 $Z$ 型异构体存在分子内氢键), 没有分离 出 $E$ 型异构体. 因此我们合成了两种常见的 $(E)-\beta$-烷基 取代脱氢氨基酸酯 $(E)-\mathbf{4 b} \sim \mathbf{4 c}$, 使用上述优化的手性单 磷配体 $(R, R)-\mathbf{3 k}$ 配体, 在优化的反应条件下对其进行了 不对称催化氢化尝试(Table 2). 结果表明 $(R, R)-3 \mathbf{k} / \mathrm{Rh}(\mathrm{I})$ 对于 $(E)-\beta$-脱氢氨基酸酯的催化不对称氢化是一种高效 的催化剂, 甲酯衍生物反应的对映选择性要优于乙酯 (Entry 1 vs Entry 2). 以 $(E)-\mathbf{4 b}$ 为底物, 将催化剂用量降 至 $0.2 \mathrm{~mol} \%$, 反应依然可以顺利进行, 产物的 $e e$ 值略有 降低(Entry 3,92\%). 对于 $\beta$ 位为乙基取代的 $(E)-\beta$-脱氢 氨基酸酯衍生物相应产物的 $e e$ 为 $96 \%$.

表 $2(R, R)-\mathbf{3 k} / \mathrm{Rh}(\mathrm{I})$ 催化的 $(E)-\beta$-脱氢氨基酸酯 $(E)-\mathbf{4 a} \sim \mathbf{4} \mathbf{c}$ 的不对称氢 化

Table 2 Asymmetric hydrogenation of $(E)-\beta$-dehydroamino acid esters $(E)-\mathbf{4 a} \sim \mathbf{4} \mathbf{c}$ under the catalysis of $(R, R)-\mathbf{3 k} / \mathrm{Rh}(\mathrm{I})$

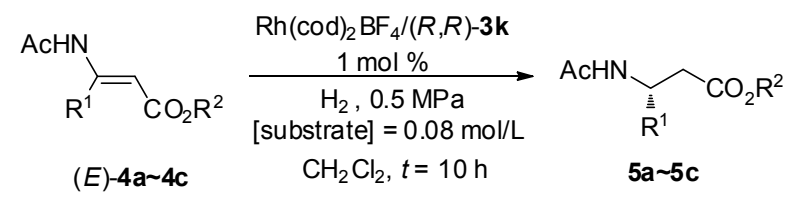

\begin{tabular}{cccccc}
\hline Entry & Substrate & $\mathrm{R}^{1}$ & $\mathrm{R}^{2}$ & Conv. $^{a} / \%$ & $e e^{b} / \%$ (Config. $^{c}$ ) \\
\hline 1 & $(E)-\mathbf{4 a}$ & $\mathrm{Me}$ & $\mathrm{Me}$ & $>99$ & $96(S)$ \\
2 & $(E)-\mathbf{4 b}$ & $\mathrm{Me}$ & $\mathrm{Et}$ & $>99$ & $94(S)$ \\
$3^{d}$ & $(E)-\mathbf{4 b}$ & $\mathrm{Me}$ & $\mathrm{Et}$ & $>99$ & $92(S)$ \\
4 & $(E)-\mathbf{4 c}$ & $\mathrm{Et}$ & $\mathrm{Me}$ & $>99$ & $96(S)$ \\
\hline
\end{tabular}

${ }^{a}$ Determined by ${ }^{1} \mathrm{H}$ NMR. ${ }^{b}$ Determined by chiral GC. ${ }^{c}$ Absolute configuration was determined by comparison of $[\alpha]_{\mathrm{D}}^{20}$ with that reported in literature. ${ }^{d}$ $n[(E)-\mathbf{4 b}]: n$ (cat) $=500: 1$.

\subsection{DpenPhos 在 $\mathrm{Rh}(\mathrm{I})$ 催化的(Z)- $\beta$-脱氢氨基酸甲酯 衍生物不对称催化氢化中的应用}

在 $\beta$-脱氢氨基酸酯的不对称催化氢化中, 对于许多 催化剂体系, $Z$ 型底物无论是在反应活性还是对映选择 性方面一般都要比相应的 $E$ 型底物低 ${ }^{[1 \mathrm{a} \sim 10 \mathrm{a}]}$, 这可能与 $Z$ 型 $\beta$-脱氢氨基酸酯底物中存在分子内氢键有关, 由于分 子内氢键的稳定化作用, 在制备相应底物时主要生成 $Z$ 型异构体, 因此探索 $Z$ 型 $\beta$-脱氢氨基酸酯的不对称催化 氢化是非常必要的. 由于 DpenPhos/Rh(I)在 $(E)-\beta$-脱氢 氨基酸酯的不对称氢化反应中展现了优秀的催化活性 和对映选择性, 因此我们进一步探索此类催化剂在 (Z)- $\beta$-脱氢氨基酸酯的不对称催化氢化反应中的应用.
首先对苯基取代的 $Z$ 型 $\beta$-脱氢氨基酸甲酯( $Z$ )-4d 的 氢化反应进行了初步尝试，篎选发现(见支持信息 (Supporting Information, SI)中 Table S1), 在与 $E$ 型 $\beta$-脱 氢氨基酸酯氢化相似的条件下，配体 $(R, R)-\mathbf{3 a} \sim \mathbf{3 h}$ 与 $\mathrm{Rh}(\mathrm{I})$ 形成的催化剂活性均较差, 以 $(R, R)-3 \mathrm{e}$ 为配体时, 在 $4 \mathrm{MPa}$ 氢气压力下, 以二氯甲烷为溶剂, 只有 $70 \%$ 的 底物转化率，但反应的对映选择性为 $87 \%$; 反应在醇类 溶剂中进行时活性较高, 虽然可以实现底物的定量转 化, 但得到的产物几乎消旋. 我们认为在醇类溶剂中之 所以能取得较高的催化活性, 这可能与底物的分子内的 氢键被醇类溶剂所影响有关，因此选择合适的混合溶剂 有可能实现反应活性和对映选择性优化 ${ }^{[10 \mathrm{a}]}$.

首先选择 $1: 2$ 二氯甲烷/异丙醇混合溶剂, 在 2 $\mathrm{MPa}$ 氢气压力和百分之一催化剂用量下, 以 $(Z)-3-$ 乙酰 胺基-2-丁烯酸甲酯( $Z$ )-4a 为模型底物, 篮选了在 $(E)-\mathbf{4 a}$ 的氢化反应中性能较好的配体 $3 \mathbf{i} \sim 30$ (Table 3, Entries $1 \sim 7)$, 在此实验条件下, 所有配体形成的催化剂均表 现出良好的催化活性, 而配体 $(R, R)-31$ 在模型底物的氢 化反应中对映选择性最好，产物 $e e$ 值达到 83\%(Entry 4). 由于该类底物的反应对溶剂比较敏感, 因此以 $(R, R)-\mathbf{3 1}$ 为配体, 在 $2 \mathrm{MPa}$ 氢气压力下, 继续对溶剂体系进行优 化, 以二氯甲烷和不同醇的混合溶剂为反应介质 (Entries 4, 8 10), 发现二氯甲烷和异丙醇的体系仍然 为最佳体系. 而只用二氯甲烷为溶剂时, 反应的活性和 选择性都有所下降(Entry 8). 进一步优化二氯甲烷与异 丙醇的比例(Entries $11 \sim 14$ ), 发现溶剂的配比对反应的 对映选择性影响很大，当二氯甲烷和异丙醇的体积比为 2：1 时, 反应的对映选择性可达到 91\% (Entry 13). 底 物的浓度对反应的对映选择性也有一定影响(Entries 13, 15 17), 在 (Z)-4a 氢化反应中, 随着底物浓度的增加, 反应的对映选择性随之增加, 当底物的浓度为 0.33 $\mathrm{mol} / \mathrm{L}$ 时, 产物的 $e e$ 值可达到 92\%(Entry 15). 但继续增 加底物浓度，则底物不能在溶剂中完全溶解。这样，通 过对配体和反应条件的篎选, 我们得到了优化的催化剂 体系和反应条件: 即以 $(R, R)-31$ 为配体, 在 $2: 1$ 二氯甲 烷/异丙醇混合溶剂中, 底物浓度为 $0.33 \mathrm{~mol} / \mathrm{L}, 2 \mathrm{MPa}$ 氢气压力下室温下反应.

我们进一步合成了一系列 $\beta$-取代的 $Z$ 型 $\beta$-脱氢氨基 酸酯衍生物, 在优化的反应条件下, 进行底物拓展. 如 Table 4 所示, 在 $\beta$-烷基取代 $Z$ 型底物的反应中, 使用 $(R, R)-3 \mathrm{l} / \mathrm{Rh}(\mathrm{I})$ 催化剂，反应的对映选择性在 $89 \% \sim 93 \%$, 随着底物中取代基 $\mathrm{R}^{1}$ 位阻的增加，产物的 $e e$ 值有所提高 (Entries 1, 3 4). 特别是对于 $\beta$-芳基取代的 $Z$ 型脱氢氨基 酸底物的氢化反应, 均能以优秀的对映异构体过量得到 相应的 $\beta$-氨基酸衍生物, 无论芳环上是吸电子(Entries 7 10)还是推电子取代基(Entries 11 12), 无论取代基的 位置在邻位(Entry 7)、间位(Entry 9)还是对位 (Entries 6, $8,10 \sim 12$ ), 相应氢化产物的 $e e$ 值均在 $96 \% \sim 98 \%$. 
表 3 DpenPhos 在 $\mathrm{Rh}(\mathrm{I})$ 催化的 $Z$ 型 $\beta$-脱氢氨基酸酯( $Z$ )-4a 不对称氢化中的应用: 配体与反应条件优化

Table 3 Application of DpenPhos in Rh(I)-catalyzed asymmetric hydrogenation of (Z)- $\beta$-dehydroamino acid ester (Z)-4a: optimization of chiral ligand and reaction conditions

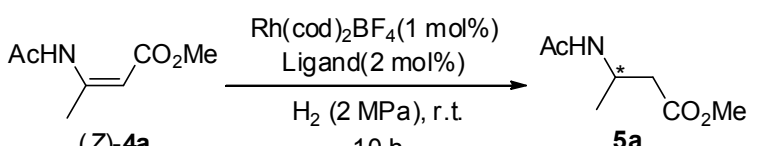

\begin{tabular}{|c|c|c|c|c|c|}
\hline Entry & Ligand & Solvent $(V: V)$ & {$[(Z)-\mathbf{4 a}] /(\mathrm{mol} / \mathrm{L})$} & Conv. ${ }^{a} / \%$ & $e e^{b} / \%$ (Config. $\left.{ }^{c}\right)$ \\
\hline 1 & $(S, S)-\mathbf{3 i}$ & $\mathrm{CH}_{2} \mathrm{Cl}_{2}: i-\mathrm{PrOH}=1: 2$ & 0.16 & $>99$ & $74(R)$ \\
\hline 2 & $(S, S)-\mathbf{3} \mathbf{j}$ & $\mathrm{CH}_{2} \mathrm{Cl}_{2}: i-\mathrm{PrOH}=1: 2$ & 0.16 & $>99$ & $65(R)$ \\
\hline 3 & $(R, R)-\mathbf{3 k}$ & $\mathrm{CH}_{2} \mathrm{Cl}_{2}: i-\mathrm{PrOH}=1: 2$ & 0.16 & $>99$ & $68(S)$ \\
\hline 4 & $(R, R)-\mathbf{3 I}$ & $\mathrm{CH}_{2} \mathrm{Cl}_{2}: i-\mathrm{PrOH}=1: 2$ & 0.16 & $>99$ & $83(S)$ \\
\hline 5 & $(S, S, R)-\mathbf{3} \mathbf{m}$ & $\mathrm{CH}_{2} \mathrm{Cl}_{2}: i-\mathrm{PrOH}=1: 2$ & 0.16 & $>99$ & $74(R)$ \\
\hline 6 & $(S, S, S)-\mathbf{3 n}$ & $\mathrm{CH}_{2} \mathrm{Cl}_{2}: i-\mathrm{PrOH}=1: 2$ & 0.16 & $>99$ & $71(R)$ \\
\hline 7 & $(R, R)-\mathbf{3 o}$ & $\mathrm{CH}_{2} \mathrm{Cl}_{2}: i-\mathrm{PrOH}=1: 2$ & 0.16 & $>99$ & $71(S)$ \\
\hline 8 & $(R, R)-\mathbf{3 l}$ & $\mathrm{CH}_{2} \mathrm{Cl}_{2}$ & 0.16 & 95 & $80(S)$ \\
\hline 9 & $(R, R)-\mathbf{3 I}$ & $\mathrm{CH}_{2} \mathrm{Cl}_{2}: n-\mathrm{PrOH}=1: 2$ & 0.16 & $>99$ & $79(S)$ \\
\hline 10 & $(R, R)-\mathbf{3 l}$ & $\mathrm{CH}_{2} \mathrm{Cl}_{2}: n-\mathrm{BuOH}=1: 2$ & 0.16 & $>99$ & $75(S)$ \\
\hline 11 & $(R, R)-\mathbf{3 l}$ & $\mathrm{CH}_{2} \mathrm{Cl}_{2}: i-\mathrm{PrOH}=1: 3$ & 0.16 & $>99$ & $72(S)$ \\
\hline 12 & $(R, R)-\mathbf{3 l}$ & $\mathrm{CH}_{2} \mathrm{Cl}_{2}: i-\mathrm{PrOH}=1: 1$ & 0.16 & $>99$ & $89(S)$ \\
\hline 13 & $(R, R)-\mathbf{3 l}$ & $\mathrm{CH}_{2} \mathrm{Cl}_{2}: i-\mathrm{PrOH}=2: 1$ & 0.16 & $>99$ & $91(S)$ \\
\hline 14 & $(R, R)-\mathbf{3 l}$ & $\mathrm{CH}_{2} \mathrm{Cl}_{2}: i-\mathrm{PrOH}=3: 1$ & 0.16 & $>99$ & $90(S)$ \\
\hline 15 & $(R, R)-\mathbf{3 I}$ & $\mathrm{CH}_{2} \mathrm{Cl}_{2}: i-\mathrm{PrOH}=2: 1$ & 0.33 & $>99$ & $92(S)$ \\
\hline 16 & $(R, R)-\mathbf{3 I}$ & $\mathrm{CH}_{2} \mathrm{Cl}_{2}: i-\mathrm{PrOH}=2: 1$ & 0.08 & $>99$ & $89(S)$ \\
\hline 17 & $(R, R)-\mathbf{3 l}$ & $\mathrm{CH}_{2} \mathrm{Cl}_{2}: i-\mathrm{PrOH}=2: 1$ & 0.05 & $>99$ & $79(S)$ \\
\hline
\end{tabular}

${ }^{a}$ Determined by ${ }^{1} \mathrm{H}$ NMR. ${ }^{b}$ Determined by chiral GC. ${ }^{c}$ Absolute configuration was determined by comparison of $[\alpha]_{0}^{20}$ with that reported in literature.

\section{3 结论}

本文研究了一类结构可调手性单齿亚磷酰胺配体 DpenPhos 在 $\mathrm{Rh}(\mathrm{I})$ 催化的 $E$ 和 $Z$ 型 $\beta$-脱氢氨基酸酯的不 对称催化氢化反应中的应用. 经过系统的反应条件和配 体结构优化, 发现 $\mathrm{N}$ 原子上含有 $\mathrm{H}$ 的亚磷酰胺配体与 $\mathrm{Rh}(\mathrm{I})$ 形成的催化剂通常比 $\mathrm{N}$ 原子上不含 $\mathrm{H}$ 的配体表现 出更高的反应活性. 使用 $(R, R)-3 \mathbf{k} / \mathrm{Rh}(\mathrm{I})$ 为催化剂, 在 3 种 $E$ 型 $\beta$-脱氢氨基酸酯的不对称氢化反应中, 取得了 $92 \% \sim 96 \%$ 的对映选择性，最低的用量可降低至 $1 / 500$; 采用 $(R, R)-3 \mathrm{l} / \mathrm{Rh}(\mathrm{I})$ 为催化剂, 在 13 种 $Z$ 型 $\beta$-脱氢氨基酸 酯不对称氢化反应中, 获得了 $92 \% \sim 98 \% e e$ 值的氢化产 物, 特别是对于 $\beta$-芳基取代衍生物的氢化反应, 相应氢 化产物的 $e e$ 值可以达到 96\% 98\%. 该类催化剂为天然 或非天然光学活性 $\beta$ 氨基酸的合成提供了一个简便、高 效的方法.

\section{4 实验部分}

\section{1 实验通则}

熔点在 XT-4 双目显微熔点测定仪测定, 温度计未 校正. ${ }^{1} \mathrm{H}$ NMR, ${ }^{31} \mathrm{P}$ NMR,${ }^{13} \mathrm{C}$ NMR 谱在 Varian Mercury 300 核磁共振仪上测定, ${ }^{1} \mathrm{H}$ NMR $\left({ }^{1} \mathrm{H} 300 \mathrm{MHz}\right)$ 的内标
表 $4(R, R)-3 \mathrm{~B} / \mathrm{Rh}(\mathrm{I})$ 催化的 $(Z)-\beta$-脱氢氨基酸酯 $(Z)-\mathbf{4 a} \sim 4 \mathbf{m}$ 的不对称氢化 Table 4 Asymmetric hydrogenation of $(Z)-\beta$-dehydroamino acid esters $(Z)-\mathbf{4 a} \sim \mathbf{4 m}$ under the catalysis of $(R, R)-\mathbf{3 l} / \mathrm{Rh}(\mathrm{I})$

[substrate] $=0.33 \mathrm{~mol} / \mathrm{L}$

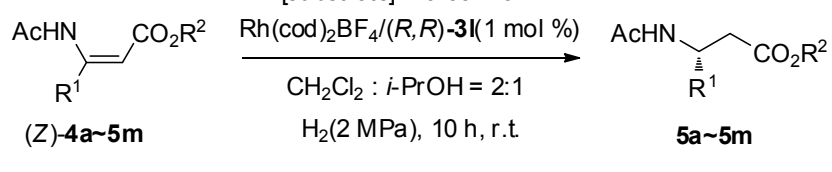

\begin{tabular}{cccccc}
\hline Entry & Substrate & $\mathrm{R}^{1}$ & $\mathrm{R}^{2}$ & Conv. $^{a}(\%)$ & $e e^{b}(\%)$ (Config. $\left.{ }^{c}\right)$ \\
\hline 1 & $(Z)-\mathbf{4 a}$ & $\mathrm{Me}$ & $\mathrm{Me}$ & $>99$ & $92(S)$ \\
2 & $(Z)-\mathbf{4 b}$ & $\mathrm{Me}$ & $\mathrm{Et}$ & $>99$ & $89(S)$ \\
3 & $(Z)-\mathbf{4 c}$ & $\mathrm{Et}$ & $\mathrm{Me}$ & $>99$ & $93(S)$ \\
4 & $(Z)-\mathbf{4 d}$ & $\mathrm{C}_{6} \mathrm{H}_{5}$ & $\mathrm{Me}$ & $>99$ & $97(R)$ \\
5 & $(Z)-\mathbf{4 e}$ & $\mathrm{C}_{6} \mathrm{H}_{5}$ & $\mathrm{Et}$ & $>99$ & $92(R)$ \\
6 & $(Z)-\mathbf{4 f}$ & $4-\mathrm{ClC}_{6} \mathrm{H}_{4}$ & $\mathrm{Me}$ & $>99$ & $97(R)$ \\
7 & $(Z)-\mathbf{4 g}$ & $2-\mathrm{ClC}_{6} \mathrm{H}_{4}$ & $\mathrm{Me}$ & $>99$ & $97(\text { n.e. })^{d}$ \\
8 & $(Z)-\mathbf{4 h}$ & $4-\mathrm{BrC}_{6} \mathrm{H}_{4}$ & $\mathrm{Me}$ & $>99$ & $98(R)$ \\
9 & $(Z)-\mathbf{4 i}$ & $3-\mathrm{BrC}_{6} \mathrm{H}_{4}$ & $\mathrm{Me}$ & $>99$ & $98(\text { n.e. })^{d}$ \\
10 & $(Z)-\mathbf{4 j}$ & $4-\mathrm{FC}_{6} \mathrm{H}_{4}$ & $\mathrm{Me}$ & $>99$ & $98(R)$ \\
11 & $(Z)-\mathbf{4 k}$ & $4-\mathrm{MeC}_{6} \mathrm{H}_{4}$ & $\mathrm{Me}$ & $>99$ & $97(R)$ \\
12 & $(Z)-\mathbf{4 l}$ & $4-\mathrm{MeOC}_{6} \mathrm{H}_{4}$ & $\mathrm{Me}$ & $>99$ & $97(R)$ \\
13 & $(Z)-\mathbf{4 m}$ & $2-\mathrm{Naphthyl}$ & $\mathrm{Me}$ & $>99$ & $96(\text { n.e. })^{d}$ \\
\hline
\end{tabular}

${ }^{a}$ Determined by ${ }^{1} \mathrm{H}$ NMR. ${ }^{b}$ Determined by chiral GC. ${ }^{c}$ Absolute configuration was determined by comparison of $[\alpha]_{\mathrm{D}}^{20}$ with that reported in literature. ${ }^{d}$ n.e. $=$ not established. 
为 $\operatorname{TMS}\left(\delta\right.$ 0.0), $\mathrm{CDCl}_{3}\left(\delta\right.$ 7.27), $\mathrm{CD}_{3} \mathrm{OD}(\delta$ 3.31) 或 $\left(\mathrm{CD}_{3}\right)_{2} \mathrm{SO}(\delta 2.50),{ }^{31} \mathrm{P} \mathrm{NMR}(121 \mathrm{MHz})$ 以 $85 \%$ 的磷酸水溶 液为外标, IR 在 Bio-Rad FIS-185 型红外光谱仪上测定. MS 在 HP5973N 和 AGILENT5973 型质谱仪上测定; HRMS(EI)在 Finnigan $\mathrm{MA}^{+}$质谱仪上测定, HRMS(ESI) 在 Kratos Concept $1 \mathrm{H}$ 质谱仪上测定, HRMS(MALDI)在 Ion Spec 4.7 Tesla FTMS 质谱仪上测定. 比旋光值在 PE-341 自动旋光仪上测定. ee 值通过 JASCO 1580 HPLC 和 Agilent $6890 \mathrm{~N} \mathrm{GC}$ 测定. 元素分析(EA)在 Elemental VARIO EL 型仪器上测定. 对空气、湿气敏感 的实验是利用手套箱和标准的 Schlenk 技术, 在氮气或 氩气氛围下操作完成. 固体商业试剂除非特别说明外, 未经纯化直接使用; 四氢呋喃、甲苯、二甲苯、乙醚、 正己烷经钠丝处理后使用; 二氯甲烷、乙腈、二甲基甲 酰胺经氢化钻干燥后使用.

\section{2 手性配体 $3 \mathbf{a} \sim 30$ 的合成}

所有单齿亚磷酰胺配体 $\mathbf{3 a} \sim 30$ 的合成均按照我们 前期报道的文献方法进行 ${ }^{[5 \sim 5 c]}$.

\section{3 底物的合成}

4.3.1 (E)-和 $(Z)$-型 $\beta$-烷基取代- $\beta$-脱氢氨基酸酯 $\mathbf{4 a} \sim \mathbf{4} \mathbf{c}$ 的合成

$(E)$-和 $(Z)-\beta$-脱氢氨基酸酯 $\mathbf{4 a} \sim \mathbf{4} \mathbf{c}$ 的合成参考文献

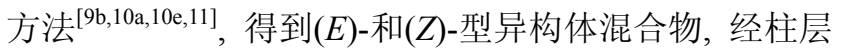
析 $(V$ 石油㜆 $: V$ 乙役乙酷 $=4: 1)$, 首先被洗脱部分为 $(Z)$ - 型产 物, 然后得到 $(E)$-型产物. 波谱数据见支持性息 $(\mathrm{SI})$.

4.3.2 (Z)-型 $\beta$-芳基取代- $\beta$-脱氢氨基酸酯 $\mathbf{4 d} \sim \mathbf{4 m}$ 的合 成

$\beta$-芳基取代- $\beta$-脱氢氨基酸酯 $4 \mathrm{~d} \sim \mathbf{4 m}$ 的合成参考上 述 $\beta$-烷基取代- $\beta$-脱氢氨基酸酯的合成方法 ${ }^{[9 b, 10 a, 100,11]}$, 但只分离到顺式产物. 已知化合物的波谱数据见补充材 料(SI), 其中 $(Z)-\mathbf{4 g}$ 和 $\mathbf{4 m}$ 为新化合物, 分析数据如下:

(Z)-4g: 48\%收率, 无色粘稠液体, ${ }^{1} \mathrm{H}$ NMR $(300$ $\left.\mathrm{MHz}, \mathrm{CDCl}_{3}\right) \delta: 10.96(\mathrm{br}, 1 \mathrm{H}), 7.23 \sim 7.37(\mathrm{~m}, 4 \mathrm{H}), 5.06$ (s, 1H), 3.78 (s, 3H), 2.13 (s, 3H); ESI-MS $(\mathrm{m} / \mathrm{z}):\left[\mathrm{M}^{+}+\right.$ $\mathrm{Na}$ ], 276.0; HRMS (ESI) calcd for $\left[\mathrm{C}_{12} \mathrm{H}_{12} \mathrm{NO}_{3} \mathrm{Cl}+\mathrm{Na}\right]^{+}$ 276.0398, found 276.0402. Anal. calcd for $\mathrm{C}_{12} \mathrm{H}_{12} \mathrm{NO}_{3} \mathrm{Cl}$ : C 56.81, H 4.77, N 5.52; found C 57.13, H 4.83, N 5.39.

(Z)-4m: 48\%收率, 白色固体, m.p. $114 \sim 115{ }^{\circ} \mathrm{C},{ }^{1} \mathrm{H}$ $\operatorname{NMR}\left(300 \mathrm{MHz}, \mathrm{CDCl}_{3}\right) \delta: 10.73(\mathrm{br}, 1 \mathrm{H}), 7.79 \sim 7.88(\mathrm{~m}$, $4 \mathrm{H}), 7.43 \sim 7.51(\mathrm{~m}, 3 \mathrm{H}), 5.41(\mathrm{~s}, 1 \mathrm{H}), 3.79(\mathrm{~s}, 3 \mathrm{H}), 2.20$ (s, 3H); ${ }^{13} \mathrm{C}$ NMR $\left(75 \mathrm{MHz}, \mathrm{CDCl}_{3}\right) \delta: 21.05,51.54$, 105.81, 114.48, 122.35, 126.53, 126.89, 127.70, 1228.74, $128.88,130.41,130.52,132.79,134.39,158.29,164.68$; ESI-MS $(\mathrm{m} / \mathrm{z})$ : $\left[\mathrm{M}^{+}+\mathrm{Na}\right], 292.1$; HRMS (ESI) calcd for $\left[\mathrm{C}_{16} \mathrm{H}_{15} \mathrm{NO}_{3}+\mathrm{Na}\right]^{+}$292.0944, found 292.0947. Anal. calcd for $\mathrm{C}_{16} \mathrm{H}_{15} \mathrm{NO}_{3}$ : C 71.36, H 5.61; found C 71.12, H 5.41 .

\section{4 催化氢化}

4.4.1 (E)-型 $\beta$-取代- $\beta$-脱氢氨基酸酯 $(E)-\mathbf{4 a} \sim \mathbf{4} \mathbf{c}$ 的氢化 反应

一般方法: 在氩气气氛中, 将体积为 $20 \mathrm{~mL}$ 的氢化 反应瓶无水无氧处理, 加入 $\mathrm{Rh}(\mathrm{cod}){ }_{2} \mathrm{BF}_{4}(2 \mathrm{mg}, 0.005$ $\mathrm{mmol})$, 再加入配体 $(R, R)-\mathbf{3 k}(5.3 \mathrm{mg}, 0.01 \mathrm{mmol})$ 及底物 $(E)-\mathbf{4 a} \sim \mathbf{4} \mathbf{c}(0.5 \mathrm{mmol})$, 置换氩气三次, 加入 $6 \mathrm{~mL}$ 二氯 甲烷, 搅拌 $10 \mathrm{~min}$, 在手套箱中将氢化瓶转移至高压釜 中, 置换氢气三次, 充入氢气至 $0.5 \mathrm{MPa}$, 室温搅拌 10 $\mathrm{h}$, 停止搅拌后，小心放掉氢气，减压除去溶剂，一部分 残余物通过测定 ${ }^{1} \mathrm{H}$ NMR, 确定底物转化率; 剩余粗产 物以乙酸乙酯为淋洗剂, 经一硅胶短柱分离, 得到氢化 产物 $5 \mathbf{a} \sim 5 \mathbf{c}$, 以配有手性柱的高效液相或气相色谱测 定其对映异构体过量.

\subsection{2 (Z)-型 $\beta$-取代- $\beta$-脱氢氨基酸酯( $Z$ ) $-\mathbf{4} \mathbf{a} \sim \mathbf{4 m}$ 的氢化 反应}

一般方法: 在氩气气氛中, 将体积为 $10 \mathrm{~mL}$ 的氢化 反应瓶无水无氧处理, 加入 $\mathrm{Rh}(\operatorname{cod}){ }_{2} \mathrm{BF}_{4}(2 \mathrm{mg}, 0.005$ $\mathrm{mmol})$, 再加入配体 $(R, R)-\mathbf{3 l}(5.8 \mathrm{mg}, 0.01 \mathrm{mmol})$, 用氩 气置换反应瓶内气体三次, 加入 $1 \mathrm{~mL}$ 二氯甲烷, 室温 下摚拌 $10 \mathrm{~min}$, 然后加入底物 $(Z)-\mathbf{4 a} \sim \mathbf{4 m}(0.5 \mathrm{mmol})$ 的 异丙醇溶液 $(0.5 \mathrm{~mL})$, 搅拌 $10 \mathrm{~min}$, 在手套箱中将氢化 瓶转移至高压釜中, 用氢气置换氞气三次, 充入氢气至 $2 \mathrm{MPa}$, 室温搅拌 $10 \mathrm{~h}$. 停止搅拌后, 小心放掉氢气, 减 压除去溶剂，一部分残余物通过测定 ${ }^{1} \mathrm{H}$ NMR，确定底 物转化率; 剩余粗产物以乙酸乙酯为淋洗剂, 经一硅胶 短柱分离, 得到氢化产物 $\mathbf{5 a} \sim 5 \mathrm{~m}$, 以配有手性柱的高 效液相或气相色谱测定其对映异构体过量. 已知产物的 物理常数与波谱数据以及 $e e$ 值测定条件见支持信息 (SI), 其中 $5 \mathrm{~g}$ 和 $5 \mathrm{~m}$ 为新化合物, 分析数据如下:

$(-)-5 \mathrm{~g}$ : 转化率: $>99 \%, 97 \% e e,[\alpha]_{\mathrm{D}}^{20}=-37.0(\mathrm{c}$ $\left.0.20, \mathrm{CHCl}_{3}\right) ;{ }^{1} \mathrm{H}$ NMR $\left(300 \mathrm{MHz}, \mathrm{CDCl}_{3}\right) \delta: 7.34 \sim 7.38$ $(\mathrm{m}, 2 \mathrm{H}), 7.20 \sim 7.25(\mathrm{~m}, 2 \mathrm{H}), 6.86(\mathrm{br}, 1 \mathrm{H}), 5.68 \sim 5.71$ (m, 1H), 3.61 (s, 3H), 2.84 3.03 (m, 2H), 2.04 (s, 3H); ${ }^{13} \mathrm{C}$ NMR $\left(75.4 \mathrm{MHz}, \mathrm{CDCl}_{3}\right) \delta: 23.28,38.07,47.59$, $51.82,126.24,126.96,127.60,127.77,128.67,128.83$, 130.04, 171.68; ESI-MS $(\mathrm{m} / \mathrm{z}):\left[\mathrm{M}^{+}+\mathrm{Na}\right], 278.1$; HRMS (ESI) calcd for $\left[\mathrm{C}_{12} \mathrm{H}_{14} \mathrm{NO}_{3} \mathrm{Cl}+\mathrm{Na}\right]^{+} 278.0554$, found 278.0565. ee 值测定条件: Chiralcel OD column; $90: 10$ $\left(V_{\text {hexane }}: V_{\text {isopropanol }}\right)$; flow rate $=1.0 \mathrm{~mL} / \mathrm{min}$; UV detection at $\lambda=230 \mathrm{~nm} ; t_{\mathrm{R}}=15.3 \mathrm{~min}$ (minor), $18.9 \mathrm{~min}$ (major).

$(-)-5 \mathrm{~m}$ : 转化率: $>99 \%, 96 \% e e,[\alpha]_{\mathrm{D}}^{20}=-100.9(\mathrm{c}$ $\left.0.55, \mathrm{CHCl}_{3}\right) ;{ }^{1} \mathrm{H}$ NMR $\left(300 \mathrm{MHz}, \mathrm{CDCl}_{3}\right) \delta: 7.72 \sim 7.80$ $(\mathrm{m}, 4 \mathrm{H}), 7.37 \sim 7.47(\mathrm{~m}, 3 \mathrm{H}), 6.87(\mathrm{br}, 1 \mathrm{H}), 5.57 \sim 5.59$ (m, 1H), $3.59(\mathrm{~s}, 3 \mathrm{H}), 2.86 \sim 3.04(\mathrm{~m}, 2 \mathrm{H}), 2.01(\mathrm{~s}, 3 \mathrm{H})$; ${ }^{13} \mathrm{C}$ NMR $\left(75.4 \mathrm{MHz}, \mathrm{CDCl}_{3}\right) \delta: 23.50,39.59,49.55$, $51.89,124.44,124.90,126.08,126.32,127.60,127.95$, 
128.61, 132.76, 133.21, 137.80, 169.37, 171.80; ESI-MS $(\mathrm{m} / \mathrm{z}): \quad\left[\mathrm{M}^{+}+\mathrm{Na}\right], 294.1 ;$ HRMS $(\mathrm{ESI})$ calcd for $\left[\mathrm{C}_{16} \mathrm{H}_{17} \mathrm{NO}_{3}+\mathrm{Na}\right]^{+}$294.1101, found 294.1103. ee 值测定 条件: Chiralcel AD-H column; 90 : 10 ( $V_{\text {hexane }}$ : $\left.V_{\text {isopropanol }}\right)$; flow rate $=1.0 \mathrm{~mL} / \mathrm{min}$; UV detection at $\lambda=$ $230 \mathrm{~nm} ; t_{\mathrm{R}}=16.1 \mathrm{~min}$ (minor), $19.4 \mathrm{~min}$ (major).

\section{References}

[1] For reviews, see: (a) Handbook of Homogeneous Hydrogenation, Vol. 1-3, Eds.: de Vries, J. G.; Elsevier, C. J., Wiley-VCH, Weinheim, 2007; (b) Shang, G.; W. Li, X. Zhang, In Catalytic Asymmetric Synthesis, 3rd ed., Ed.: Ojima, I., Wiley-Blackwell, Hoboken, 2010, pp. $343-436$.

[2] (a) Reetz, M. T.; Mehler, G. Angew. Chem. Int. Ed. 2000, 39, 3889; (b) van den Berg, M.; Minnaard, A. J.; Schudde, E. P.; van Esch, J.; de Vries, A. H. M.; de Vries, J. G.; Feringa, B. L. J. Am. Chem. Soc. 2000, 122, 11539; (c) Claver, C.; Fernandez, E.; Gillon, A.; Heslop, K.; Hyett, D. J.; Martorell, A.; Orpen, A. G.; Pringle, P. G. Chem. Commun. 2000, 961. For reviews: (d) Minnaard, A. J.; Feringa, B. L.; Lefort, L.; De Vries, J. G. Acc. Chem. Res. 2007, 40, 1267; (e) Bruneau, C.; Renaud, J.-L. in Phosporus Ligands in Asymmetric Catalysis: Synthesis and Applications, Ed.: Börner, A., Wiley-VCH, Weinheim, 2008, pp. 36-69.

[3] (a) Komarov, I. V.; Börner, A. Angew. Chem. Int. Ed. 2001, 40, 1197; (b) Guo, H. C.; Ding, K.; Dai, L.-X. Chin. Sci. Bull. 2004, 49, 2003. (c) Teichert, J. F.; Feringa, B. L. Angew. Chem.. Int. Ed. 2010, 49, 2486; (d) Reetz, M. T. Angew. Chem. Int. Ed. 2008, 47, 2256; (e) Zhang, Z. F.; Xie, F.; Yang, B.; Yu, H.; Zhang, W. B. Chin. J. Org. Chem. 2011, 31, 429 (in Chinese). (张振峰, 谢芳, 杨波, 余焓, 张 万斌, 有机化学, 2011, 31, 429.)

[4] (a) Fu, Y.; Xie, J.-H.; Hu, A.-G.; Zhou, H.; Wang, L.-X.; Zhou, Q.-L. Chem. Commun. 2002, 480; (b) Hu, A.-G.; Fu, Y.; Xie, J.-H.; Zhou, H.; Wang, L.-X.; Zhou, Q.-L. Angew. Chem. Int. Ed. 2002, 41, 2348.

[5] (a) Liu, Y.; Ding, K. J. Am. Chem. Soc. 2005, 127, 10488; (b) Liu, Y.; Sandoval, C. A.; Yamaguchi, Y.; Zhang, X.; Wang, Z.; Kato, K.; Ding, K. J. Am. Chem. Soc. 2006, 128, 14212; (c) Zhang, J. Z.; Li, Y.; Wang, Z.; Ding, K. Angew. Chem. Int. Ed. 2011, 50, 11743; (d) Zhang, J. Z.; Dong, K.; Wang, Z.; Ding, K. Org. Biomol. Chem. 2011, 11, 1598 .

[6] (a) Gademann, K.; Hintermann, T.; Schreiber, J. V. Curr. Med. Chem. 1999, 6, 905; (b) von Nussbaum, F.; Spiteller, P. In Highlights in Bioorganic Chemistry: Methods and Application, Eds.: Schmuck, C.; Wennemers, H., Wiley-VCH, Weinheim, 2004, p. 63.

[7] For reviews, see: (a) Enantioselective Synthesis of $\beta$-Amino Acids,
Eds.: Juaristi, E.; Soloshnok, V., John Wiley \& Sons, Inc., Hoboken, 2005; (b) Weiner, B.; Szymański, W.; Janssen, D. B.; Minnaard, A. J.; Feringa, B. L. Chem. Soc. Rev. 2010, 39, 1656; (c) Ma, Y.-H.; Zhang, Y.-J.; Zhang, W.-B. Chin. J. Org. Chem. 2007, 27, 289. (马元 辉, 张勇健, 张万斌, 有机化学, 2007, 27, 289.)

[8] For early examples, see: (a) Achiwa, K.; Soga, T. Tetrahedron Lett. 1978, 13, 1119; (b) Lubell, W. D.; Kitamura, M.; Noyori, R. Tetrahedron: Asymmetry 1991, 2, 543; (c) Zhu, G.; Chen, Z.; Zhang, X. J. Org. Chem. 1999, 64, 6907.

[9] For examples, see: (a) Liu, D.; Zhang, X. Eur. J. Org. Chem. 2005, 646; (b) Tang, W.; Wang, W.; Chi, Y.; Zhang, X. Angew. Chem. Int. Ed. 2003, 41, 3509; (c) Zhou, Y. G.; Tang, W.; Wang, W. B.; Li, W.; Zhang, X. J. Am. Chem. Soc. 2002, 124, 4952; (d) Yasutake, M.; Gridnev, I. D.; Higashi, N.; Imamoto, T. Org. Lett. 2001, 3, 1701; (e) Wu, H. P.; Hoge, G. Org. Lett. 2004, 6, 3645; (f) Lee, S.-G.; Zhang, Y. J. Org. Lett. 2002, 4, 2429; (g) Hu, X. P.; Zheng, Z. Org. Lett. 2005, 7, 419; (h) You, J.; Drexler, H. J.; Zhang, S.; Fischer, C.; Heller, D. Angew. Chem. Int. Ed. 2003, 42, 913; (i) Wu, J.; Chen, X.; Guo, R.; Yeung, C.-H.; Chan, A. S. C. J. Org. Chem. 2003, 68, 2490; (j) Tang, W.; Wu, S.; Zhang, X. J. Am. Chem. Soc. 2003, 125, 9570; (k) Hsiao, Y.; Rivera, N. R.; Rosner, T.; Krska, S. W.; Njolito, E.; Wang, F.; Sun, Y.; Armstrong, III, J. D.; Grabowski, E. J. J.; Tillyer, R. D.; Spindler, F; Malan, C. J. Am. Chem. Soc. 2004, 126, 9918; (1) Dai, Q.; Yang W.; Zhang, X. Org. Lett. 2005, 7, 5343; (m) Qiu, L.; Wu, J.; Chan, S.; Au-Yeung, T. T.-L.; Ji, J.-X.; Guo, R.; Pai, C.-C.; Zhou, Z.; Li, X.; Fan, Q.-H.; Chan, A. C. S. Proc. Natl. Acad. Sci. U. S. A. 2004, 101, 5815; (n) Qiu, L.; Kwong, F. Y.; Wu, J.; Lam, W. H.; Chan, S.; Yu, W.-Y.; Li, Y.-M.; Guo, R.; Zhou Z.; Chan, A. C. S J. Am. Chem. Soc. 2006, 128, 5955; (o) Huang, H.; Liu, X.; Deng, J.; Qiu, M.; Zheng, Z. Org. Lett. 2006, 8, 3359; (p) Qiu, L.; Prashad, M.; Hu, B.; Prasad, K.; Repiĉ, O.; Blacklock, T. J.; Kwong, F. Y.; Kok, S. H. L.; Lee, H. W.; Chan, A. S. C. Proc. Natl. Acad. Sci. U. S A. 2007, 104, 16787; (q) Deng, J.; Hu, X.-P.; Huang, J.-D.; Yu, S.-B.; Wang, D.-Y.; Duan, Z.-C.; Zheng, Z. J. Org. Chem. 2008, 73, 2015.

[10] For examples, see: (a) Peña, D.; Minnaard, A. J.; de Vries, J. G; Feringa, B. L. J. Am. Chem. Soc. 2002, 124, 14552; (b) Reetz, M. T.; Li, X. Tetrahedron 2004, 60, 9709; (c) Lefort, L.; Boogers, J. A. F.; de Vreis, A. H. M.; De Vreis, J. G.; Org. Lett. 2004, 6, 1733; (d) Reetz, M. T.; Li, X. Angew. Chem. Int. Ed. 2005, 44, 2959; (e) Fu, Y.; Guo, X. X.; Zhou, S. F.; Hu, A. G.; Xie, J. H.; Zhou, Q. L. J. Org. Chem. 2004, 69, 4648; (f) Fu, Y.; Hou, G. H.; Xie, J. H.; Xing, L.; Wang, L. X.; Zhou, Q. L. J. Org. Chem. 2004, 69, 8157; (g) Huang, H. M.; Zheng, Z.; Luo, H. L.; Bai, C. M.; Hu, X.; Chen, H. L. J. Org. Chem. 2004, 69, 2335.

[11] Bernsmann, H.; V. D. Berg, M.; Hoen, R.; Minnaard, A. J.; Reetz, M. T.; Mehler, G.; de Vries, J. G.; Feringa, B. L. J. Org. Chem. 2005 $70,943$. 